\title{
Observation of Single Top-Quark Production
}

V. M. Abazov,${ }^{36}$ B. Abbott,${ }^{74}$ M. Abolins,${ }^{64}$ B. S. Acharya,${ }^{29}$ M. Adams,${ }^{50}$ T. Adams,${ }^{48}$ E. Aguilo, ${ }^{6}$ M. Ahsan,${ }^{58}$ G. D. Alexeev, ${ }^{36}$ G. Alkhazov, ${ }^{40}$ A. Alton, ${ }^{64}, *$ G. Alverson, ${ }^{62}$ G. A. Alves, ${ }^{2}$ L. S. Ancu, ${ }^{35}$ T. Andeen, ${ }^{52}$ M. S. Anzelc, ${ }^{52}$

M. Aoki ${ }^{49}$ Y. Arnoud,${ }^{14}$ M. Arov,${ }^{59}$ M. Arthaud ${ }^{18}$ A. Askew ${ }^{48, \dagger}$ B. Asman, ${ }^{41}$ O. Atramentov, ${ }^{48, \dagger}$ C. Avila, ${ }^{8}$

J. BackusMayes ${ }^{81}$ F. Badaud, ${ }^{13}$ L. Bagby ${ }^{49}$ B. Baldin, ${ }^{49}$ D. V. Bandurin,${ }^{58}$ P. Banerjee, ${ }^{29}$ S. Banerjee,${ }^{29}$ E. Barberis, ${ }^{62}$ A.-F. Barfuss, ${ }^{15}$ P. Bargassa, ${ }^{79}$ P. Baringer ${ }^{57}$ J. Barreto, ${ }^{2}$ J. F. Bartlett, ${ }^{49}$ U. Bassler ${ }^{18}$ D. Bauer, ${ }^{43}$ S. Beale, ${ }^{6}$ A. Bean,${ }^{57}$

M. Begalli, ${ }^{3}$ M. Begel,${ }^{72}$ C. Belanger-Champagne,${ }^{41}$ L. Bellantoni, ${ }^{49}$ A. Bellavance, ${ }^{49}$ J. A. Benitez, ${ }^{64}$ S. B. Beri, ${ }^{27}$

G. Bernardi, ${ }^{17}$ R. Bernhard, ${ }^{23}$ I. Bertram, ${ }^{42}$ M. Besançon, ${ }^{18}$ R. Beuselinck, ${ }^{43}$ V. A. Bezzubov, ${ }^{39}$ P. C. Bhat, ${ }^{49}$

V. Bhatnagar, ${ }^{27}$ G. Blazey, ${ }^{51}$ S. Blessing, ${ }^{48}$ K. Bloom, ${ }^{66}$ A. Boehnlein, ${ }^{49}$ D. Boline, ${ }^{61}$ T. A. Bolton,${ }^{58}$ E. E. Boos, ${ }^{38}$ G. Borissov, ${ }^{42}$ T. Bose,${ }^{76}$ A. Brandt, ${ }^{77}$ R. Brock, ${ }^{64}$ G. Brooijmans, ${ }^{69}$ A. Bross, ${ }^{49}$ D. Brown, ${ }^{19}$ X. B. Bu, ${ }^{7}$ N. J. Buchanan ${ }^{48}$ D. Buchholz, ${ }^{52}$ M. Buehler, ${ }^{80}$ V. Buescher, ${ }^{22}$ V. Bunichev, ${ }^{38}$ S. Burdin, ${ }^{42,}$ T. H. Burnett, ${ }^{81}$ C. P. Buszello, ${ }^{43}$ P. Calfayan, ${ }^{25}$ B. Calpas, ${ }^{15}$ S. Calvet, ${ }^{16}$ J. Cammin, ${ }^{70}$ M. A. Carrasco-Lizarraga, ${ }^{33}$ E. Carrera,${ }^{48}$ W. Carvalho, ${ }^{3}$ B. C. K. Casey, ${ }^{49}$ H. Castilla-Valdez, ${ }^{33}$ S. Chakrabarti, ${ }^{71}$ D. Chakraborty, ${ }^{51}$ K. M. Chan,${ }^{54}$ A. Chandra, ${ }^{47}$ E. Cheu, ${ }^{45}$ D. K. Cho, ${ }^{61}$ S. Choi, ${ }^{32}$ B. Choudhary, ${ }^{28}$ L. Christofek, ${ }^{76}$ T. Christoudias, ${ }^{43}$ S. Cihangir, ${ }^{49}$ D. Claes, ${ }^{66}$ J. Clutter,${ }^{57}$ Y. Coadou, ${ }^{6,8}$ M. Cooke, ${ }^{49}$

W. E. Cooper ${ }^{49}$ M. Corcoran,${ }^{79}$ F. Couderc, ${ }^{18}$ M.-C. Cousinou, ${ }^{15}$ S. Crépé-Renaudin, ${ }^{14}$ V. Cuplov, ${ }^{58}$ D. Cutts, ${ }^{76}$

M. Ćwiok, ${ }^{30}$ A. Das, ${ }^{45}$ G. Davies,${ }^{43}$ K. De ${ }^{77}$ S. J. de Jong,${ }^{35}$ E. De La Cruz-Burelo, ${ }^{33}$ K. DeVaughan, ${ }^{66}$ F. Déliot,,${ }^{18}$ M. Demarteau, ${ }^{49}$ R. Demina, ${ }^{70}$ D. Denisov, ${ }^{49}$ S. P. Denisov, ${ }^{39}$ S. Desai, ${ }^{49}$ H. T. Diehl, ${ }^{49}$ M. Diesburg, ${ }^{49}$ A. Dominguez, ${ }^{66}$ T. Dorland, ${ }^{81}$ A. Dubey, ${ }^{28}$ L. V. Dudko, ${ }^{38}$ L. Duflot, ${ }^{16}$ D. Duggan, ${ }^{48}$ A. Duperrin, ${ }^{15}$ S. Dutt, ${ }^{27}$ A. Dyshkant, ${ }^{51}$ M. Eads,${ }^{66}$ D. Edmunds, ${ }^{64}$ J. Ellison, ${ }^{47}$ V. D. Elvira, ${ }^{49}$ Y. Enari, ${ }^{76}$ S. Eno, ${ }^{60}$ P. Ermolov, ${ }^{38,|l| l}$ M. Escalier, ${ }^{15}$ H. Evans, ${ }^{53}$

A. Evdokimov, ${ }^{72}$ V. N. Evdokimov, ${ }^{39}$ A. V. Ferapontov ${ }^{58}$ T. Ferbel,${ }^{61,70}$ F. Fiedler, ${ }^{24}$ F. Filthaut, ${ }^{35}$ W. Fisher,${ }^{49}$ H. E. Fisk ${ }^{49}$ M. Fortner ${ }^{51}$ H. Fox,${ }^{42}$ S. Fu, ${ }^{49}$ S. Fuess, ${ }^{49}$ T. Gadfort, ${ }^{69}$ C. F. Galea ${ }^{35}$ A. Garcia-Bellido, ${ }^{70}$ V. Gavrilov,${ }^{37}$ P. Gay,${ }^{13}$ W. Geist ${ }^{19}$ W. Geng, ${ }^{15,64}$ C. E. Gerber, ${ }^{50}$ Y. Gershtein,${ }^{48, \dagger}$ D. Gillberg, ${ }^{6}$ G. Ginther ${ }^{70}$ B. Gómez, ${ }^{8}$ A. Goussiou, ${ }^{81}$ P. D. Grannis, ${ }^{71}$ S. Greder,${ }^{19}$ H. Greenlee, ${ }^{49}$ Z. D. Greenwood, ${ }^{59}$ E. M. Gregores, ${ }^{4}$ G. Grenier, ${ }^{20}$ Ph. Gris, ${ }^{13}$ J.-F. Grivaz, ${ }^{16}$ A. Grohsjean, ${ }^{25}$ S. Grünendahl, ${ }^{49}$ M. W. Grünewald,${ }^{30}$ F. Guo,${ }^{71}$ J. Guo,${ }^{71}$ G. Gutierrez,${ }^{49}$ P. Gutierrez, ${ }^{74}$ A. Haas, ${ }^{69}$ N. J. Hadley, ${ }^{60}$ P. Haefner, ${ }^{25}$ S. Hagopian, ${ }^{48}$ J. Haley,${ }^{67}$ I. Hall,${ }^{64}$ R. E. Hall,${ }^{46}$ L. Han, ${ }^{7}$ K. Harder ${ }^{44}$ A. Harel,${ }^{70}$ J. M. Hauptman ${ }^{56}$ J. Hays ${ }^{43}$ T. Hebbeker, ${ }^{21}$ D. Hedin, ${ }^{51}$ J. G. Hegeman, ${ }^{34}$ A. P. Heinson, ${ }^{47}$ U. Heintz, ${ }^{61}$ C. Hensel,,${ }^{22, \|}$ K. Herner, ${ }^{63}$ G. Hesketh, ${ }^{62}$ M. D. Hildreth, ${ }^{54}$ R. Hirosky, ${ }^{80}$ T. Hoang, ${ }^{48}$ J. D. Hobbs, ${ }^{71}$ B. Hoeneisen, ${ }^{12}$ M. Hohlfeld, ${ }^{22}$ S. Hossain, ${ }^{74}$ P. Houben, ${ }^{34}$ Y. Hu, ${ }^{71}$ Z. Hubacek, ${ }^{10}$ N. Huske, ${ }^{17}$ V. Hynek, ${ }^{10}$ I. Iashvili, ${ }^{68}$ R. Illingworth, ${ }^{49}$ A. S. Ito, ${ }^{49}$ S. Jabeen, ${ }^{61}$ M. Jaffré ${ }^{16}$ S. Jain, ${ }^{74}$ K. Jakobs,${ }^{23}$ D. Jamin,${ }^{15}$ C. Jarvis, ${ }^{60}$ R. Jesik ${ }^{43}$ K. Johns, ${ }^{45}$ C. Johnson, ${ }^{69}$ M. Johnson, ${ }^{49}$ D. Johnston, ${ }^{66}$ A. Jonckheere, ${ }^{49}$ P. Jonsson, ${ }^{43}$ A. Juste, ${ }^{49}$ E. Kajfasz, ${ }^{15}$ D. Karmanov, ${ }^{38}$ P. A. Kasper, ${ }^{49}$ I. Katsanos, ${ }^{66}$ V. Kaushik,${ }^{77}$ R. Kehoe ${ }^{78}$ S. Kermiche, ${ }^{15}$ N. Khalatyan, ${ }^{49}$ A. Khanov, ${ }^{75}$ A. Kharchilava, ${ }^{68}$ Y. N. Kharzheev, ${ }^{36}$ D. Khatidze, ${ }^{69}$ T. J. Kim, ${ }^{31}$ M. H. Kirby, ${ }^{52}$ M. Kirsch, ${ }^{21}$ B. Klima, ${ }^{49}$ J. M. Kohli, ${ }^{27}$ J.-P. Konrath, ${ }^{23}$ A. V. Kozelov, ${ }^{39}$

J. Kraus, ${ }^{64}$ T. Kuhl, ${ }^{24}$ A. Kumar, ${ }^{68}$ A. Kupco, ${ }^{11}$ T. Kurča, ${ }^{20}$ V. A. Kuzmin, ${ }^{38}$ J. Kvita, ${ }^{9}$ F. Lacroix, ${ }^{13}$ D. Lam, ${ }^{54}$

S. Lammers, ${ }^{53}$ G. Landsberg, ${ }^{76}$ P. Lebrun, ${ }^{20}$ W. M. Lee, ${ }^{49}$ A. Leflat,${ }^{38}$ J. Lellouch, ${ }^{17,\|l\|}$ J. Li,${ }^{77}$ L. Li,${ }^{47}$ Q. Z. Li, ${ }^{49}$ S. M. Lietti, ${ }^{5}$ J. K. Lim, ${ }^{31}$ D. Lincoln, ${ }^{49}$ J. Linnemann, ${ }^{64}$ V. V. Lipaev,${ }^{39}$ R. Lipton, ${ }^{49}$ Y. Liu,${ }^{7}$ Z. Liu, ${ }^{6}$ A. Lobodenko, ${ }^{40}$ M. Lokajicek, ${ }^{11}$ P. Love, ${ }^{42}$ H. J. Lubatti, ${ }^{81}$ R. Luna-Garcia, ${ }^{33,}$, A. L. Lyon, ${ }^{49}$ A. K. A. Maciel, ${ }^{2}$ D. Mackin, ${ }^{79}$ P. Mättig, ${ }^{26}$

A. Magerkurth, ${ }^{63}$ P. K. Mal, ${ }^{81}$ H. B. Malbouisson, ${ }^{3}$ S. Malik, ${ }^{66}$ V. L. Malyshev,${ }^{36}$ Y. Maravin, ${ }^{58}$ B. Martin, ${ }^{14}$

R. McCarthy, ${ }^{71}$ C. L. McGivern, ${ }^{57}$ M. M. Meijer ${ }^{35}$ A. Melnitchouk, ${ }^{65}$ L. Mendoza, ${ }^{8}$ P. G. Mercadante, ${ }^{5}$ M. Merkin, ${ }^{38}$ K. W. Merritt, ${ }^{49}$ A. Meyer, ${ }^{21}$ J. Meyer, ${ }^{22, \|}$ J. Mitrevski, ${ }^{69}$ R. K. Mommsen,${ }^{44}$ N. K. Mondal, ${ }^{29}$ R. W. Moore, ${ }^{6}$ T. Moulik,${ }^{57}$ G. S. Muanza, ${ }^{15}$ M. Mulhearn ${ }^{69}$ O. Mundal, ${ }^{22}$ L. Mundim, ${ }^{3}$ E. Nagy, ${ }^{15}$ M. Naimuddin, ${ }^{49}$ M. Narain,${ }^{76}$ H. A. Neal, ${ }^{63}$ J. P. Negret, ${ }^{8}$ P. Neustroev, ${ }^{40}$ H. Nilsen, ${ }^{23}$ H. Nogima, ${ }^{3}$ S. F. Novaes, ${ }^{5}$ T. Nunnemann, ${ }^{25}$ D. C. O'Neil, ${ }^{6}$ G. Obrant, ${ }^{40}$ C. Ochando, ${ }^{16}$ D. Onoprienko, ${ }^{58}$ J. Orduna, ${ }^{33}$ N. Oshima, ${ }^{49}$ N. Osman, ${ }^{43}$ J. Osta,${ }^{54}$ R. Otec,,${ }^{10}$ G. J. Otero y Garzón, ${ }^{1}$

M. Owen, ${ }^{44}$ M. Padilla, ${ }^{47}$ P. Padley, ${ }^{79}$ M. Pangilinan, ${ }^{76}$ N. Parashar, ${ }^{55}$ S.-J. Park, ${ }^{22, \| l}$ S. K. Park, ${ }^{31}$ J. Parsons, ${ }^{69}$

R. Partridge,${ }^{76}$ N. Parua,${ }^{53}$ A. Patwa,${ }^{72}$ G. Pawloski, ${ }^{79}$ B. Penning,${ }^{23}$ M. Perfilov,${ }^{38}$ K. Peters,${ }^{44}$ Y. Peters,${ }^{44}$ P. Pétroff, ${ }^{16}$ R. Piegaia, ${ }^{1}$ J. Piper, ${ }^{64}$ M.-A. Pleier, ${ }^{22}$ P. L. M. Podesta-Lerma, ${ }^{33, * *}$ V. M. Podstavkov, ${ }^{49}$ Y. Pogorelov, ${ }^{54}$ M.-E. Pol, ${ }^{2}$ P. Polozov, ${ }^{37}$ A. V. Popov,${ }^{39}$ C. Potter, ${ }^{6}$ W. L. Prado da Silva, ${ }^{3}$ H. B. Prosper, ${ }^{48}$ S. Protopopescu, ${ }^{72}$ J. Qian, ${ }^{63}$ A. Quadt, ${ }^{22, \|}$

B. Quinn, ${ }^{65}$ A. Rakitine, ${ }^{42}$ M. S. Rangel, ${ }^{16}$ K. Ranjan, ${ }^{28}$ P. N. Ratoff, ${ }^{42}$ P. Renkel, ${ }^{78}$ P. Rich,${ }^{44}$ M. Rijssenbeek ${ }^{71}$

I. Ripp-Baudot, ${ }^{19}$ F. Rizatdinova ${ }^{75}$ S. Robinson, ${ }^{43}$ R. F. Rodrigues, ${ }^{3}$ M. Rominsky, ${ }^{74}$ C. Royon,${ }^{18}$ P. Rubinov, ${ }^{49}$ R. Ruchti, ${ }^{54}$ G. Safronov,${ }^{37}$ G. Sajot, ${ }^{14}$ A. Sánchez-Hernández, ${ }^{33}$ M. P. Sanders,,${ }^{17}$ B. Sanghi,${ }^{49}$ G. Savage,${ }^{49}$ L. Sawyer, ${ }^{59}$ 
T. Scanlon, ${ }^{43}$ D. Schaile, ${ }^{25}$ R. D. Schamberger, ${ }^{71}$ Y. Scheglov, ${ }^{40}$ H. Schellman, ${ }^{52}$ T. Schliephake, ${ }^{26}$ S. Schlobohm, ${ }^{81}$ C. Schwanenberger ${ }^{44}$ R. Schwienhorst, ${ }^{64}$ J. Sekaric, ${ }^{48}$ H. Severini, ${ }^{74}$ E. Shabalina,${ }^{50}$ M. Shamim, ${ }^{58}$ V. Shary, ${ }^{18}$ A. A. Shchukin, ${ }^{39}$ R. K. Shivpuri, ${ }^{28}$ V. Siccardi, ${ }^{19}$ V. Simak,${ }^{10}$ V. Sirotenko, ${ }^{49}$ P. Skubic, ${ }^{74}$ P. Slattery, ${ }^{70}$ D. Smirnov, ${ }^{54}$ G. R. Snow, ${ }^{66}$ J. Snow, ${ }^{73}$ S. Snyder ${ }^{72}$ S. Söldner-Rembold, ${ }^{44}$ L. Sonnenschein, ${ }^{21}$ A. Sopczak,${ }^{42}$ M. Sosebee, ${ }^{77}$ K. Soustruznik, ${ }^{9}$ B. Spurlock, ${ }^{77}$ J. Stark, ${ }^{14}$ V. Stolin, ${ }^{37}$ D. A. Stoyanova, ${ }^{39}$ J. Strandberg, ${ }^{63}$ S. Strandberg, ${ }^{41}$ M. A. Strang, ${ }^{68}$ E. Strauss, ${ }^{71}$ M. Strauss,${ }^{74}$ R. Ströhmer, ${ }^{25}$ D. Strom,${ }^{52}$ L. Stutte, ${ }^{49}$ S. Sumowidagdo, ${ }^{48}$ P. Svoisky, ${ }^{35}$ M. Takahashi, ${ }^{44}$ A. Tanasijczuk, ${ }^{1}$ W. Taylor, ${ }^{6}$ B. Tiller, ${ }^{25}$ F. Tissandier, ${ }^{13}$ M. Titov, ${ }^{18}$ V. V. Tokmenin, ${ }^{36}$ I. Torchiani, ${ }^{23}$ D. Tsybychev, ${ }^{71}$ B. Tuchming, ${ }^{18}$ C. Tully, ${ }^{67}$ P. M. Tuts, ${ }^{69}$ R. Unalan, ${ }^{64}$ L. Uvarov ${ }^{40}$ S. Uvarov ${ }^{40}$ S. Uzunyan, ${ }^{51}$ B. Vachon, ${ }^{6}$ P. J. van den Berg, ${ }^{34}$ R. Van Kooten, ${ }^{53}$ W. M. van Leeuwen, ${ }^{34}$ N. Varelas, ${ }^{50}$ E. W. Varnes, ${ }^{45}$ I. A. Vasilyev, ${ }^{39}$ P. Verdier, ${ }^{20}$ L. S. Vertogradov, ${ }^{36}$ M. Verzocchi, ${ }^{49}$ D. Vilanova, ${ }^{18}$ P. Vint, ${ }^{43}$ P. Vokac,${ }^{10}$ M. Voutilainen,${ }^{66, \dagger}$ R. Wagner, ${ }^{67}$ H. D. Wahl,${ }^{48}$ M. H. L. S. Wang, ${ }^{49}$ J. Warchol,${ }^{54}$ G. Watts, ${ }^{81}$ M. Wayne, ${ }^{54}$ G. Weber, ${ }^{24}$ M. Weber $,{ }^{49,}, \$$ L. Welty-Rieger, ${ }^{53}$ A. Wenger, ${ }^{23,}, \S$ M. Wetstein, ${ }^{60}$ A. White ${ }^{77}$ D. Wicke, ${ }^{26}$ M. R. J. Williams, ${ }^{42}$ G. W. Wilson, ${ }^{57}$ S. J. Wimpenny, ${ }^{47}$ M. Wobisch,${ }^{59}$ D. R. Wood, ${ }^{62}$ T. R. Wyatt, ${ }^{44}$ Y. Xie,${ }^{76}$ C. Xu,${ }^{63}$ S. Yacoob,${ }^{52}$ R. Yamada, ${ }^{49}$ W.-C. Yang, ${ }^{44}$ T. Yasuda, ${ }^{49}$ Y. A. Yatsunenko, ${ }^{36}$ Z. Ye, ${ }^{49}$ H. Yin, ${ }^{7}$ K. Yip,${ }^{72}$ H. D. Yoo, ${ }^{76}$ S. W. Youn,${ }^{52}$ J. Yu, ${ }^{77}$ C. Zeitnitz, ${ }^{26}$ S. Zelitch ${ }^{80}$ T. Zhao, ${ }^{81}$ B. Zhou, ${ }^{63}$ J. Zhu, ${ }^{71}$ M. Zielinski, ${ }^{70}$ D. Zieminska, ${ }^{53}$ L. Zivkovic, ${ }^{69}$ V. Zutshi, ${ }^{51}$ and E. G. Zverev ${ }^{38}$

\title{
(D0 Collaboration)
}

\author{
${ }^{1}$ Universidad de Buenos Aires, Buenos Aires, Argentina \\ ${ }^{2}$ LAFEX, Centro Brasileiro de Pesquisas Físicas, Rio de Janeiro, Brazil \\ ${ }^{3}$ Universidade do Estado do Rio de Janeiro, Rio de Janeiro, Brazil \\ ${ }^{4}$ Universidade Federal do ABC, Santo André, Brazil \\ ${ }^{5}$ Instituto de Física Teórica, Universidade Estadual Paulista, São Paulo, Brazil \\ ${ }^{6}$ University of Alberta, Edmonton, Alberta, Canada; \\ Simon Fraser University, Burnaby, British Columbia, Canada; \\ York University, Toronto, Ontario, Canada \\ and McGill University, Montreal, Quebec, Canada \\ ${ }^{7}$ University of Science and Technology of China, Hefei, People's Republic of China \\ ${ }^{8}$ Universidad de los Andes, Bogotá, Colombia \\ ${ }^{9}$ Center for Particle Physics, Charles University, Faculty of Mathematics and Physics, Prague, Czech Republic \\ ${ }^{10}$ Czech Technical University in Prague, Prague, Czech Republic \\ ${ }^{11}$ Center for Particle Physics, Institute of Physics, Academy of Sciences of the Czech Republic, Prague, Czech Republic \\ ${ }^{12}$ Universidad San Francisco de Quito, Quito, Ecuador \\ ${ }^{13}$ LPC, Université Blaise Pascal, CNRS/IN2P3, Clermont, France \\ ${ }^{14}$ LPSC, Université Joseph Fourier Grenoble 1, CNRS/IN2P3, Institut National Polytechnique de Grenoble, Grenoble, France \\ ${ }^{15}$ CPPM, Aix-Marseille Université, CNRS/IN2P3, Marseille, France \\ ${ }^{16}$ LAL, Université Paris-Sud, IN2P3/CNRS, Orsay, France \\ ${ }^{17}$ LPNHE, IN2P3/CNRS, Universités Paris VI and VII, Paris, France \\ ${ }^{18}$ CEA, Irfu, SPP, Saclay, France \\ ${ }^{19}$ IPHC, Université de Strasbourg, CNRS/IN2P3, Strasbourg, France \\ ${ }^{20} I P N L$, Université Lyon 1, CNRS/IN2P3, Villeurbanne, France and Université de Lyon, Lyon, France \\ ${ }^{21}$ III. Physikalisches Institut A, RWTH Aachen University, Aachen, Germany \\ ${ }^{22}$ Physikalisches Institut, Universität Bonn, Bonn, Germany \\ ${ }^{23}$ Physikalisches Institut, Universität Freiburg, Freiburg, Germany \\ ${ }^{24}$ Institut für Physik, Universität Mainz, Mainz, Germany \\ ${ }^{25}$ Ludwig-Maximilians-Universität München, München, Germany \\ ${ }^{26}$ Fachbereich Physik, University of Wuppertal, Wuppertal, Germany \\ ${ }^{27}$ Panjab University, Chandigarh, India \\ ${ }^{28}$ Delhi University, Delhi, India \\ ${ }^{29}$ Tata Institute of Fundamental Research, Mumbai, India \\ ${ }^{30}$ University College Dublin, Dublin, Ireland \\ ${ }^{31}$ Korea Detector Laboratory, Korea University, Seoul, Korea \\ ${ }^{32}$ SungKyunKwan University, Suwon, Korea \\ ${ }^{33}$ CINVESTAV, Mexico City, Mexico \\ ${ }^{34}$ FOM-Institute NIKHEF and University of Amsterdam/NIKHEF, Amsterdam, The Netherlands \\ ${ }^{35}$ Radboud University Nijmegen/NIKHEF, Nijmegen, The Netherlands \\ ${ }^{36}$ Joint Institute for Nuclear Research, Dubna, Russia
}




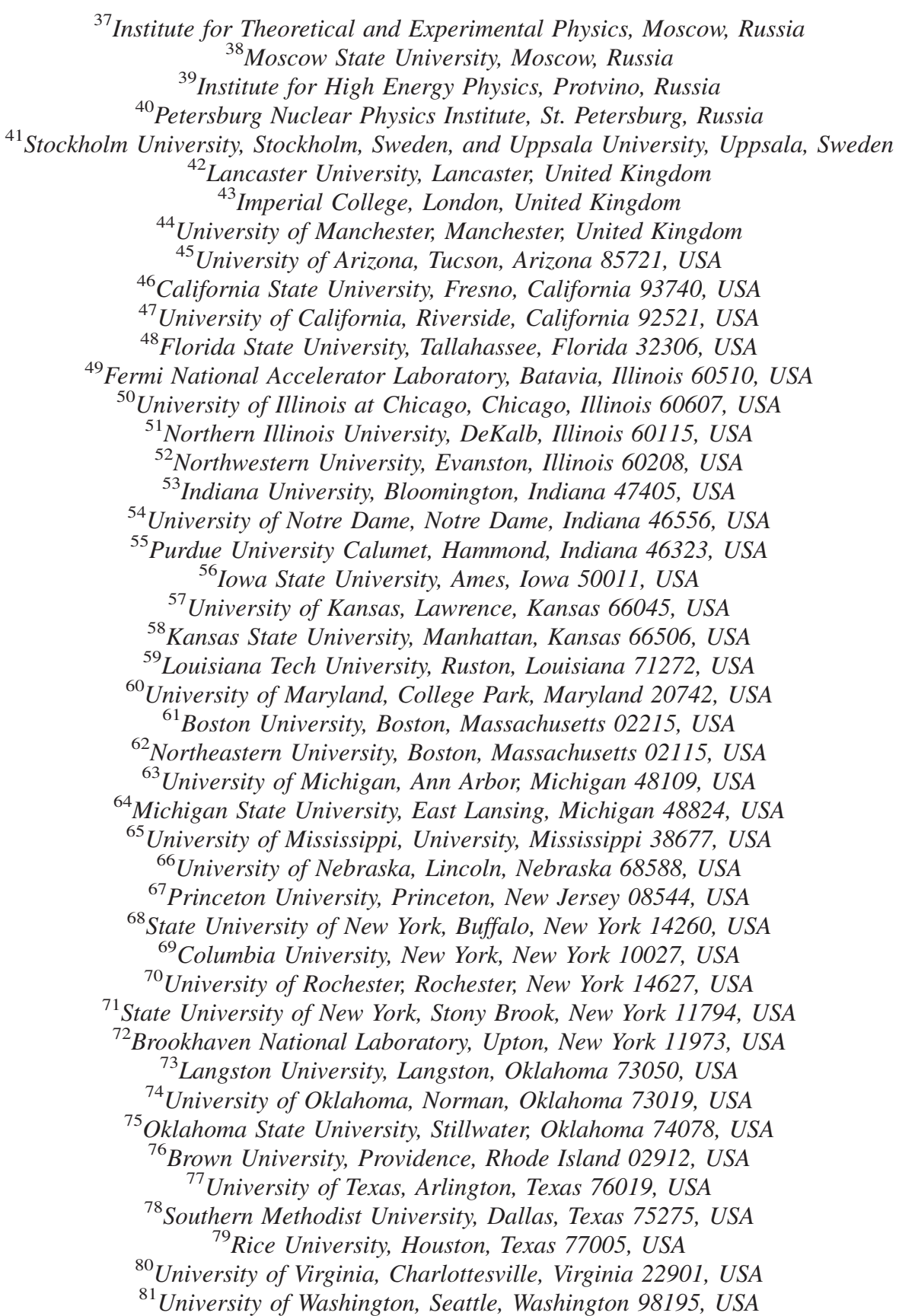

(Received 4 March 2009; revised manuscript received 10 April 2009; published 24 August 2009)

We report observation of the electroweak production of single top quarks in $p \bar{p}$ collisions at $\sqrt{s}=$ $1.96 \mathrm{TeV}$ based on $2.3 \mathrm{fb}^{-1}$ of data collected by the D0 detector at the Fermilab Tevatron Collider. Using events containing an isolated electron or muon and missing transverse energy, together with jets originating from the fragmentation of $b$ quarks, we measure a cross section of $\sigma(p \bar{p} \rightarrow t b+X, t q b+$ $X)=3.94 \pm 0.88 \mathrm{pb}$. The probability to measure a cross section at this value or higher in the absence of signal is $2.5 \times 10^{-7}$, corresponding to a 5.0 standard deviation significance for the observation.

DOI: 10.1103/PhysRevLett.103.092001

At hadron colliders, top quarks can be produced in pairs via the strong interaction or singly via the electroweak interaction [1]. Top quarks were first observed via pair production at the Fermilab Tevatron Collider in 1995 [2].
PACS numbers: 14.65.Ha, 12.15.Hh, 12.15.Ji, 13.85.Qk

Since then, pair production has been used to make precise measurements of several top-quark properties, including the top-quark mass [3]. Single top-quark production, on the other hand, serves as a probe of the Wtb interaction [4], 
and its production cross section provides a direct measurement of the magnitude of the quark mixing matrix element $V_{t b}$ without assuming three quark generations [5]. However, measuring the yield of single top quarks is difficult because of the small production rate and large backgrounds.

In 2007, we presented the first evidence for single topquark production and the first direct measurement of $\left|V_{t b}\right|$ [6] using $0.9 \mathrm{fb}^{-1}$ of Tevatron data at a center-of-mass energy of $1.96 \mathrm{TeV}$. This Letter describes the observation of a single top quark signal in $2.3 \mathrm{fb}^{-1}$ of data. The CDF collaboration recently also reported such evidence in $2.2 \mathrm{fb}^{-1}$ of data [7] and is concurrently reporting observation in $3.2 \mathrm{fb}^{-1}$ of data [8].

When top quarks are produced singly, they are accompanied by a bottom quark in the $s$-channel production mode [9] or by both a bottom quark and a light quark in $t$-channel production $[10,11]$. We search for both of these processes at once. The sum of their predicted cross sections is $3.46 \pm 0.18 \mathrm{pb}$ [11] for a top-quark mass $m_{t}=$ $170 \mathrm{GeV}$, at which this analysis is performed. We refer to the $s$-channel process as " $t b$ " production and the $t$-channel process as " $t q b$. ."

The analysis presented in this Letter is an improved version of the one from 2007 [6], with a larger data set. The data were collected with the D0 detector [12] using a logical OR of many trigger conditions in place of only the single-lepton plus jets triggers used previously. Several offline selection criteria, including $b$-jet identification requirements for double-tagged events, have been loosened. These improvements have increased the signal acceptance by $18 \%$. The backgrounds are $W$ bosons produced in association with jets, top-quark pair $(t \bar{t})$ production with decay into the lepton + jets and dilepton final states (when a jet or a lepton is not reconstructed), and multijet production, where a jet is misreconstructed as an electron or a heavy-flavor quark decays to a muon that passes isolation criteria. $Z+$ jets and diboson processes form minor additional background components.

We consider events with two, three, or four jets (which allows for additional jets from initial-state and final-state radiation), reconstructed using a cone algorithm in $(y, \phi)$ space, where $y$ is the rapidity and $\phi$ is the azimuthal angle, and the cone radius is 0.5 [6]. The highest- $p_{T}$ (leading) jet must have $p_{T}>25 \mathrm{GeV}$, and subsequent jets have $p_{T}>$ $15 \mathrm{GeV}$; all jets have pseudorapidity $|\eta|<3$.4. We require $20<\mathbb{E}_{T}<200 \mathrm{GeV}$ for events with two jets and $25<$ $\not_{T}<200 \mathrm{GeV}$ for events with three or four jets. Events must contain only one isolated electron with $p_{T}>15 \mathrm{GeV}$ and $|\eta|<1.1$ ( $p_{T}>20 \mathrm{GeV}$ for three- or four-jet events), or one isolated muon with $p_{T}>15 \mathrm{GeV}$ and $|\eta|<2.0$. The background from multijets events is kept to $\approx 5 \%$ by requiring high total transverse energy and by demanding that the $\mathbb{E}_{T}$ is not along the direction of the lepton or the leading jet. To enhance the signal fraction, one or two of the jets are required to originate from long-lived $b$ hadrons using a neural network (NN) $b$-jet tagging algorithm [13]. After $b$-jet identification, we require the leading $b$-tagged jet to have $p_{T}>20 \mathrm{GeV}$. To further improve the sensitivity, we split the data by lepton flavor, number of jets, and $b$-tagged jets, and data collection period.

We model the signal using the SINGLETOP event generator [14] interfaced to PYTHIA [15]. We assume the SM prediction for the ratio of the $t b$ and $t q b$ cross sections [11]. The $t \bar{t}, W+$ jets, and $Z+$ jets backgrounds are simulated using the ALPGEN leading-log MC event generator [16] and PYTHIA to model hadronization. The $t \bar{t}$ background is normalized to the predicted cross section [17]. The diboson backgrounds are modeled using PYTHIA. In the simulation of the $W+$ jets backgrounds, we scale the ALPGEN cross sections for events with heavy-flavor jets by factors derived from calculations of next-to-leading-order effects [18]: $W b \bar{b}$ and $W c \bar{c}$ are scaled by 1.47 , and $W c j$ by 1.38 .

All MC events are passed through a GEANT-based simulation of the D0 detector and are reconstructed using the same software as for the data. Small differences between data and simulation in the lepton and jet reconstruction efficiencies and resolutions are corrected in the simulation as measured from separate data samples. We also correct the $\eta$ (jets), $\Delta \phi$ (jet1, jet2), and $\Delta \eta$ (jet1, jet2) distributions in the $W+$ jets samples to match data. The multijets background is modeled using independent data samples containing leptons that are not isolated. The multijets background, combined with the background from $W+$ jets, is normalized to the lepton + jets data with other backgrounds subtracted, using the $p_{T}(\ell), \mathbb{E}_{T}$, and the $W$ boson transverse mass distributions before $b$-jet identification is applied.

The $b$-tagging algorithm is modeled in simulated events by applying weights ("tag-rate functions") measured from data that account for the probability for each jet to be tagged as a function of jet flavor, $p_{T}$, and $\eta$. After $b$ tagging, an empirical correction of $0.95 \pm 0.13$ for the $W b \bar{b}$ and $W c \bar{c}$ fractions is derived from the $b$-tagged and not- $b$-tagged two-jet data and simulated samples.

The above selections give $4519 b$-tagged lepton + jets events, which are expected to contain $223 \pm 30$ single topquark events. Table I shows the event yields, separated by jet multiplicity. The percentage acceptances are (3.7 \pm $0.5) \%$ for $t b$ and $(2.5 \pm 0.3) \%$ for $t q b$.

Systematic uncertainties arise from each correction factor or function applied to the background and signal models. Most affect only the normalization, but three corrections modify in addition the shapes of the distributions; these are the jet energy scale corrections, the tag-rate functions, and the reweighting of the distributions in $W+$ jets events. The largest uncertainties come from the jet energy scale [the normalization part is $(1.1-13.1) \%$ for signal and $(0.1-2.1) \%$ for background], the tag-rate func- 
TABLE I. Number of expected and observed events in $2.3 \mathrm{fb}^{-1}$ for $e$ and $\mu$, and 1 and $2 b$-tagged analysis channels combined. The uncertainties include both statistical and systematic components.

\begin{tabular}{lccc}
\hline \hline \multicolumn{1}{c}{ Source } & 2 jets & 3 jets & 4 jets \\
\hline$t b+t q b$ signal & $139 \pm 18$ & $63 \pm 10$ & $21 \pm 5$ \\
$W+$ jets & $1829 \pm 161$ & $637 \pm 61$ & $180 \pm 18$ \\
$Z+$ jets and dibosons & $229 \pm 38$ & $85 \pm 17$ & $26 \pm 7$ \\
$t \bar{t}$ & $222 \pm 35$ & $436 \pm 66$ & $484 \pm 71$ \\
Multijets & $196 \pm 50$ & $73 \pm 17$ & $30 \pm 6$ \\
Total prediction & $2615 \pm 192$ & $1294 \pm 107$ & $742 \pm 80$ \\
Data & 2579 & 1216 & 724 \\
\hline \hline
\end{tabular}

tions [the normalization part is $(2.1-7.0) \%$ for singletagged events and (9.0-11.4)\% for double-tagged events], and the correction for jet-flavor composition in $W+$ jets events $(13.7 \%)$, with smaller contributions from the integrated luminosity $(6.1 \%)$, jet energy resolution $(4.0 \%)$, initial-state and final-state radiation $(0.6 \%-12.6 \%), b$-jet fragmentation (2.0\%), tz cross section (12.7\%), and lepton efficiency corrections (2.5\%). All other contributions have a smaller effect. The values given are the relative uncertainties on the individual sources. The total uncertainty on the background is (8-16)\% depending on the analysis channel.

After event selection, we expect single top-quark events to constitute (3-9)\% of the data sample. Since the uncertainty on the background is larger than the expected signal, we improve discrimination by using multivariate analysis techniques. We have developed three independent analyses based on boosted decision trees (BDT) [19], Bayesian neural networks (BNN) [20], and the matrix element (ME) method [21]. Our application of these techniques to D0's single top-quark searches is described in Ref. [6]. The analyses presented in this Letter differ from previous implementations in the choice of input variables and some detailed tuning of each technique.

The BDT analysis has reoptimized the input variables [22] into a common set of 64 variables for all analysis channels. The variables fall into five categories, singleobject kinematics, global event kinematics, jet reconstruction, top-quark reconstruction, and angular correlations. Separate sets of trees are created with these variables for each channel. The BNN analysis uses the RuleFitJF algorithm [23] to select the most sensitive of these variables, then combines 18-28 of them into a single separate discriminant for each channel. The ME analysis uses only twojet and three-jet events, divided into a $W+$ jets-dominated set and a $t \bar{t}$-dominated set. It includes matrix elements for more background sources, adding $t \bar{t}, W W, W Z$, and $g g g$ diagrams in the two-jet bin and Wugg in the three-jet bin, to improve background rejection.

Each analysis uses the same data and background model and has the same sources of systematic uncertainty. We test the analyses using ensembles of pseudo data sets created from background and signal at different cross sections to confirm linear behavior and thus an unbiased cross section measurement. The analyses are also checked extensively before $b$-tagging is applied, and using two control regions of the data, one dominated by $W+$ jets and the other by $t \bar{t}$ backgrounds, as shown in Fig. 1. These studies confirm that backgrounds are well modeled across the full range of the discriminant output.

The cross section is determined using the same Bayesian approach as in our previous studies [6]. This involves forming a binned likelihood as a product over all bins and channels, evaluated separately for each multivariate discriminant, with no cuts applied to the outputs. The central value of the cross section is defined by the position of the peak in the posterior density, and the 68\% interval about the peak is taken as the uncertainty on the measurement. Systematic uncertainties, including all correlations, are reflected in this posterior interval.

We extract inclusive single top-quark cross sections $\sigma(p \bar{p} \rightarrow t b+X, t q b+X) \quad$ of $\quad \sigma_{\mathrm{BDT}}=3.74_{-0.79}^{+0.95} \mathrm{pb}$, $\sigma_{\mathrm{BNN}}=4.70_{-0.93}^{+1.18} \mathrm{pb}$, and $\sigma_{\mathrm{ME}}=4.30_{-1.20}^{+0.99} \mathrm{pb}$. The sensitivity of the analyses to a contribution from single topquark production is estimated by generating an ensemble of pseudo data sets that sample the background model and its uncertainties, with no signal present. We measure a cross section from each pseudo data set, and hence obtain the probability that the SM cross section is reached. This provides expected sensitivities [stated in terms of Gaussian standard deviations, (SD)] of 4.3, 4.1, and 4.1 SD for the BDT, BNN, and ME analyses, respectively. The measured significances, obtained by counting the number of pseudo data sets above the measured cross section, are 4.6, 5.2, and 4.9 SD, respectively.

The three multivariate techniques use the same data sample but are not completely correlated: the correlation of the measured cross section using pseudo data sets with background and SM signal is BDT : $\mathrm{BNN}=74 \%$, $\mathrm{BDT}: \mathrm{ME}=60 \%, \mathrm{BNN}: \mathrm{ME}=57 \%$. Their combination therefore leads to increased sensitivity and a more precise measurement of the cross section. We use the three discriminant outputs as inputs to a second set of Bayesian
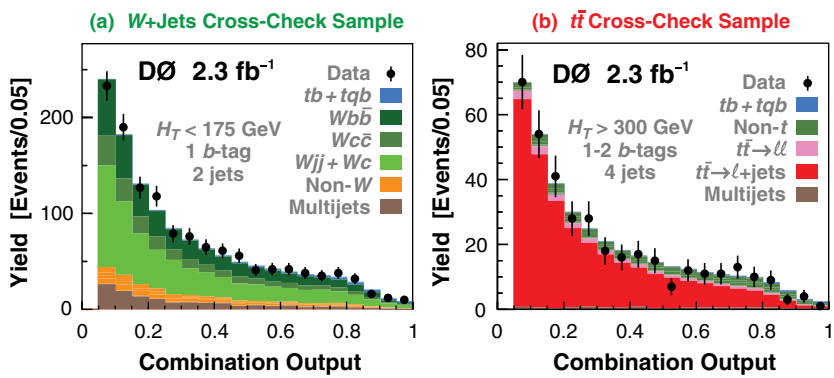

FIG. 1 (color online). The combination discriminant outputs for (a) $W+$ jets and (b) $t \bar{t}$ cross-check samples. $H_{T}$ is the scalar sum of the $p_{T}$ of the final-state objects (lepton, $\mathbb{E}_{T}$, and jets). 

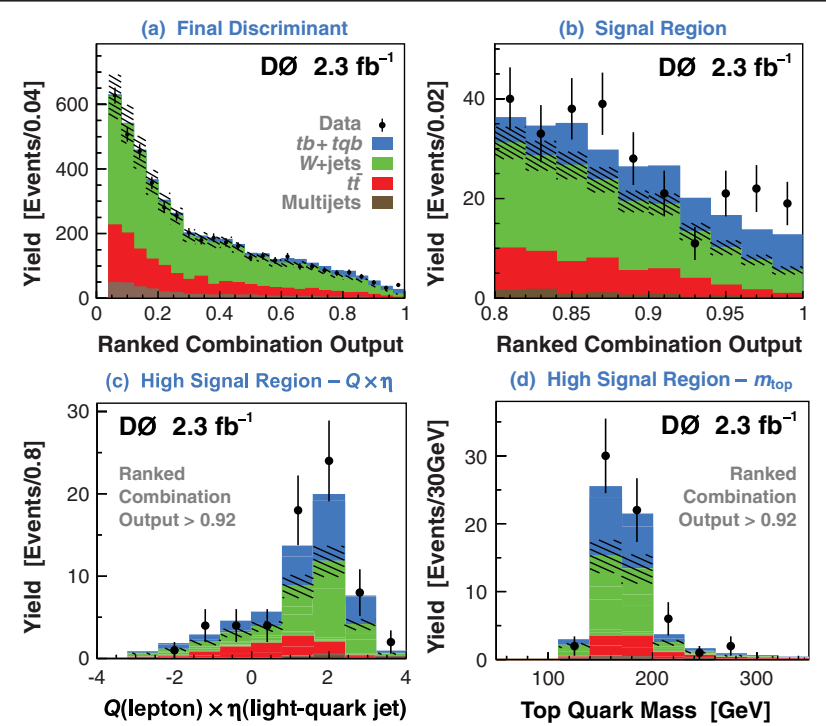

(d) High Signal Region $-m_{\text {top }}$

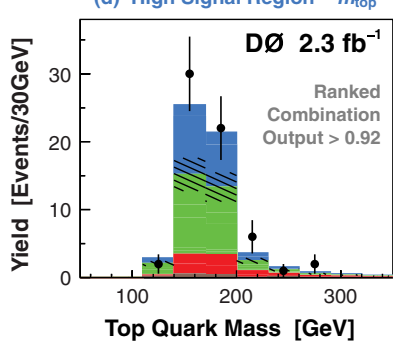

FIG. 2 (color online). Distribution of the combination output for all 24 analysis channels combined, (a) full range, and (b) high signal region. The bins have been ordered by their expected signal to background ratio and the signal is normalized to the measured cross section. The hatched band indicates the total uncertainty on the background. For the ranked combination output $>0.92$, (c) shows the distribution of lepton charge times pseudorapidity of the leading not- $b$-tagged jet, and (d) shows the reconstructed top-quark mass.

neural networks, and obtain the combined cross section and its signal significance from the new discriminant output. The resulting expected significance is 4.5 SD. Figure 2 illustrates the importance of the signal when comparing data to prediction. The measured cross section is $\sigma(p \bar{p} \rightarrow$ $t b+X, t q b+X)=3.94 \pm 0.88 \mathrm{pb}$.

The measurement has a $p$ value of $2.5 \times 10^{-7}$, corresponding to a significance of 5.0 SD. The expected and measured posterior densities and the background-only pseudodataset measurements are shown in Fig. 3.

We use the cross section measurement to determine the Bayesian posterior for $\left|V_{t b}\right|^{2}$ in the interval $[0,1]$ and ex-
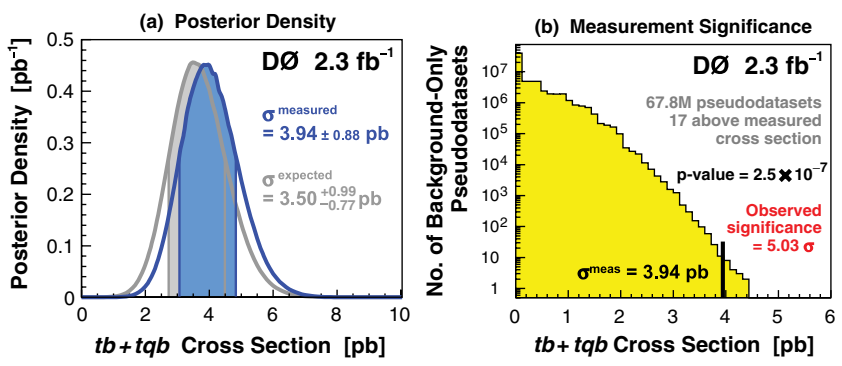

FIG. 3 (color online). (a) Expected SM and measured Bayesian posterior probability densities for the $t b+t q b$ cross section. The shaded regions indicate 1 standard deviation above and below the peak positions. (b) Measured cross sections using the ensemble of background-only pseudo data sets (containing full systematics and no signal) used to measured the significance of the result. tract a limit of $\left|V_{t b}\right|>0.78$ at $95 \%$ C.L. within the SM [6]. When the upper constraint is removed, we measure $\left|V_{t b} f_{1}^{L}\right|=1.07 \pm 0.12$, where $f_{1}^{L}$ is the strength of the left-handed $W t b$ coupling.

In summary, we have measured the single top-quark production cross section using $2.3 \mathrm{fb}^{-1}$ of data at the D0 experiment. We measure a cross section for the combined $t b+t q b$ channels of $3.94 \pm 0.88 \mathrm{pb}$. Our result provides an improved direct measurement of $V_{t b}$. The measured single top-quark signal corresponds to an excess over the predicted background with a significance of 5.0 SD-observation of single top-quark production.

We thank the staffs at Fermilab and collaborating institutions, and acknowledge support from the DOE and NSF (USA); CEA and CNRS/IN2P3 (France); FASI, Rosatom and RFBR (Russia); CNPq, FAPERJ, FAPESP and FUNDUNESP (Brazil); DAE and DST (India); Colciencias (Colombia); CONACyT (Mexico); KRF and KOSEF (Korea); CONICET and UBACyT (Argentina); FOM (The Netherlands); STFC (United Kingdom); MSMT and GACR (Czech Republic); CRC Program, CFI, NSERC and WestGrid Project (Canada); BMBF and DFG (Germany); SFI (Ireland); The Swedish Research Council (Sweden); CAS and CNSF (China); and the Alexander von Humboldt Foundation (Germany).

*Visitor from Augustana College, Sioux Falls, SD, USA.

${ }^{\dagger}$ Visitor from Rutgers University, Piscataway, NJ, USA.

${ }^{*}$ Visitor from The University of Liverpool, Liverpool, United Kingdom.

${ }^{\S}$ Visitor from CERN, Geneva, Switzerland.

"Visitor from II. Physikalisches Institut, Georg-AugustUniversity, Göttingen, Germany.

"Visitor from Centro de Investigacion en Computacion IPN, Mexico City, Mexico.

**Visitor from ECFM, Universidad Autonoma de Sinaloa, Culiacán, Mexico.

${ }^{\dagger \dagger}$ Visitor from Helsinki Institute of Physics, Helsinki, Finland.

$\$$ Visitor from Universität Bern, Bern, Switzerland.

${ }^{\S}$ Visitor from Universität Zürich, Zürich, Switzerland.

${ }^{\|l\|}$ Deceased.

[1] S. S. D. Willenbrock and D. A. Dicus, Phys. Rev. D 34, 155 (1986).

[2] F. Abe et al. (CDF Collaboration), Phys. Rev. Lett. 74, 2626 (1995); S. Abachi et al. (D0 Collaboration), Phys. Rev. Lett. 74, 2632 (1995).

[3] C. Amsler et al., (Particle Data Group), Phys. Lett. B 667, 1 (2008).

[4] A. P. Heinson et al. Phys. Rev. D 56, 3114 (1997); V. M. Abazov et al. (D0 Collaboration), Phys. Rev. Lett. 101, 221801 (2008).

[5] G. V. Jikia and S. R. Slabospitsky, Phys. Lett. B 295, 136 (1992). 
[6] V. M. Abazov et al. (D0 Collaboration), Phys. Rev. Lett. 98, 181802 (2007); V.M. Abazov et al. (D0) Collaboration), Phys. Rev. D 78, 012005 (2008).

[7] T. Aaltonen et al. (CDF Collaboration), Phys. Rev. Lett. 101, 252001 (2008).

[8] T. Aaltonen et al. (CDF Collaboration), following Letter, Phys. Rev. Lett. 103, 092002 (2009).

[9] S. Cortese and R. Petronzio, Phys. Lett. B 253, 494 (1991).

[10] C.-P. Yuan, Phys. Rev. D 41, 42 (1990).

[11] N. Kidonakis, Phys. Rev. D 74, 114012 (2006). Individual cross sections: $\sigma_{t b}=1.12 \pm 0.05 \mathrm{pb}$ and $\sigma_{t q b}=2.34 \pm$ $0.13 \mathrm{pb}$.

[12] V.M. Abazov et al. (D0 Collaboration), Nucl. Instrum. Methods Phys. Res., Sect. A 565, 463 (2006).

[13] T. Scanlon, Ph.D. thesis, Imperial College, University of London, 2006.

[14] E. E. Boos et al., Phys. At. Nucl. 69, 1317 (2006). We used SINGLETOP version $4.2 \mathrm{p} 1$.

[15] T. Sjöstrand, S. Mrenna, and P. Skands, J. High Energy Phys. 05 (2006) 026. We used PYTHIA version 6.409.
[16] M. L. Mangano et al., J. High Energy Phys. 07 (2003) 001. We used ALPGEN version 2.11.

[17] N. Kidonakis and R. Vogt, Phys. Rev. D 68, 114014 (2003). We used $\sigma(p \bar{p} \rightarrow t \bar{t}+X)=7.91_{-1.01}^{+0.61} \mathrm{pb}$, including an uncertainty component for the top-quark mass.

[18] J. M. Campbell and R. K. Ellis, Phys. Rev. D 65, 113007 (2002).

[19] L. Breiman et al., Classification and Regression Trees (Wadsworth, Stamford, 1984); J. A. Benitez, Ph.D. thesis, Michigan State University, 2009; D. Gillberg, Ph.D. thesis, Simon Fraser University, 2009.

[20] R. M. Neal, Bayesian Learning for Neural Networks (Springer-Verlag, New York, 1996); A. Tanasijczuk, Ph.D. thesis, Universidad de Buenos Aires, 2009.

[21] V. M. Abazov et al. (D0 Collaboration), Nature (London) 429, 638 (2004); M. Pangilinan, Ph.D. thesis, Brown University, 2009.

[22] Q.-H. Cao et al. Phys. Rev. D 71, 054023 (2005); Q.-H. Cao et al., Phys. Rev. D 72, 094027 (2005).

[23] J. H. Friedman and B. E. Popescu, Ann. Appl. Stat. 2, 916 (2008). 